\title{
The relationship between destination proximity, destination mix and physical activity behaviors
}

\author{
Gavin R. McCormack*, Billie Giles-Corti, Max Bulsara \\ School of Population Health, The University of Western Australia, 35 Stirling Highway, Crawley, Western Australia, 6009, Australia
}

Available online 8 February 2007

\begin{abstract}
Background. The presence and mix of destinations is an important aspect of the built environment that may encourage or discourage physical activity. This study examined the association between the proximity and mix of neighbourhood destinations and physical activity.

Methods. Secondary analysis was undertaken on physical activity data from Western Australian adults $(n=1394)$. These data were linked with geographical information systems (GIS) data including the presence and the mix of destinations located within 400 and $1500 \mathrm{~m}$ from respondents' homes. Associations with walking for transport and recreation and vigorous physical activity were examined.

Results. Access to post boxes, bus stops, convenience stores, newsagencies, shopping malls, and transit stations within 400 m (OR 1.63-5.00) and schools, transit stations, newsagencies, convenience stores and shopping malls within $1500 \mathrm{~m}$ (OR 1.75-2.38) was associated with participation in regular transport-related walking. A dose-response relationship between the mix of destinations and walking for transport was also found. Each additional destination within 400 and $1500 \mathrm{~m}$ resulted in an additional 12 and $11 \mathrm{~min} /$ fortnight spent walking for transport, respectively.

Conclusion. Proximity and mix of destinations appears strongly associated with walking for transport, but not walking for recreation or vigorous activity. Increasing the diversity of destinations may contribute to adults doing more transport-related walking and achieving recommended levels of physical activity.
\end{abstract}

(C) 2007 Elsevier Inc. All rights reserved.

Keywords: Environment; Exercise; Physical activity; Walking

\section{Background}

The built environment provides opportunities and barriers to physical activity participation (Humpel et al., 2002; McCormack et al., 2004; Owen et al., 2004; Saelens et al., 2003b). Land development patterns and urban sprawl in particular have resulted in increased distances between homes and destinations, lower density suburbs, and disconnected street patterns. These environmental features result in fewer transport-related walking trips, fewer recreational walking trips and less recreational physical activity (Frank and Engelke, 2001; Frank et al., 2003).

Studies have found associations between proximity of destinations and physical activity. For example, high levels of spatial access to attractive public open space and to the beach appear to be positively associated with both walking for recreation and walking for transport (Giles-Corti and Donovan,

\footnotetext{
* Corresponding author.

E-mail address: gavinm@dph.uwa.edu.au (G.R. McCormack).
}

2002b, 2003) while poor spatial access to built recreational facilities appears to decrease participation in physical activity (Giles-Corti and Donovan, 2002a). Transport-related walking is also negatively associated with distance to community rail-trails (Troped et al., 2003). Exercise-related walking within the neighbourhood (Humpel et al., 2004) and overall levels of physical activity (Bauman et al., 1999) are found to be higher among residents living in coastal compared with inland postal code districts. Moreover, positive associations between land-use mix, density of local destinations and physical activity have also been found (Handy, 1996; Handy and Clifton, 2001b; Hoehner et al., 2005; Sallis et al., 1990).

Of particular importance in studies examining the relationship between land-use and physical activity is the definition of a 'walkable' distance. Generally, walkable distances have ranged from $90 \mathrm{~m}$ to $1 \mathrm{~km}$ (Cervero and Radisch, 1996; Frank, 2004; Hoehner et al., 2005; Pikora et al., 2002; Sallis et al., 1990) or a 10 - to 15 min walk from home (Saelens et al., 2003a). AultmanHall et al. (1997) suggest that $400 \mathrm{~m}$ is considered the greatest 
distance a transit walker is likely to walk to a transit station. Recent Western Australian Liveable Neighborhood Guidelines, which promote development of destinations within a walkable distance of 400 to $450 \mathrm{~m}$ reflect this suggestion (The Government of Western Australia, 2000).

To date, distances to recreational and transport-related destinations have been shown to influence their use and physical activity. However, the associations between specific physical activity behaviors and road network distance from home to specific destinations have not been examined. This study involved the secondary analyses of data collected as part of the Study of Environmental and Individual Determinants of Physical Activity (SEID 1) (Giles-Corti and Donovan, 2002a). The aims of this study were: (1) to examine the association between walking for transport, walking for recreation, and vigorous physical activity and the presence of specific destinations within 400 and $1500 \mathrm{~m}$ of respondent's homes and (2) to examine the association between these same physical activities and the mix of destinations located within 400 and $1500 \mathrm{~m}$ of respondent's homes.

\section{Methods}

\section{Sample}

SEID 1 involved a cross-section of adults aged 18 to 59 years $(n=1803)$ from a $408 \mathrm{~km}^{2}$ area of Perth, Western Australia (Giles-Corti and Donovan, 2002a). Data collection commenced in late spring 1995 and took 5 months to complete. It involved face-to-face interviews in the respondents' homes with a follow-up telephone survey $(n=1474)$ undertaken 2 to 4 weeks later. Respondents were recruited from households located in 277 census collectors districts (CD) from the 80th (137 CDs and $n=929)$ and 20th (140 CDs and $n=874$ ) percentiles of social disadvantage. The recruitment of the first household within each $\mathrm{CD}$ was determined by overlaying a network grid onto a CD map, and using random numbers to select a starting point. The recruitment of households thereafter was based on a skip interval, which provided each household located within a $\mathrm{CD}$ an equal chance of being selected. One respondent per household was randomly selected. The response rates for the baseline and follow-up surveys were $52.9 \%$ and $81.8 \%$, respectively. This study only includes data from those who participated in both surveys. The University of Western Australia Human Rights Committee granted ethics approval.

\section{Dependent variables}

\section{Physical activity}

In the baseline and follow-up survey respondents reported their frequency and duration of walking for recreation, walking for transport, and vigorous physical activity, in the past 2 weeks. The physical activity items are reliable and valid in the Australian context (Australian Health and Welfare, 2003; Booth et al., 1996). Specifically, these items have shown acceptable test-retest reliability $(\mathrm{ICC}=0.40-0.68)$.

To capture habitual physical activity data from both surveys were used. Two physical activity outcome variables, each for walking for recreation, walking for transport and vigorous physical activity were derived. Firstly, a trichotomous outcome variable was developed for each activity that included inactivity (i.e., no activity reported at either the baseline or follow-up survey), irregular participation (i.e., activity reported at only one survey), and regular participation (i.e., activity reported at both surveys), regardless of frequency or time spent participating. Secondly, among regular and irregular active respondents, a continuous outcome variable was developed for each activity of average time/ fortnight spent participating in the activity. This was calculated using the reported time from both surveys (i.e., duration at baseline plus duration at follow-up divided by two).

\section{Independent variables}

\section{Destinations present}

Destination data were determined using GIS, with street address information derived from the Yellow Pages Telephone Directory, the White Pages Telephone Directory, the Australian postal service (Australia Post), the Western Australian Department of Transport, and the Western Australian Ministry of Planning. The shortest road network distance to utilitarian trip-related $(n=8)$ and recreational $(n=3)$ destinations including: shops, post boxes, convenience stores, newsagents, schools, bus stops, transit stations, parks, the river, and beaches within the Perth metropolitan area was used. For each destination, two variables were derived: (1) destination present within $400 \mathrm{~m}$ (i.e., 437.4 yards) of home and (2) destination present within $1500 \mathrm{~m}$ (i.e., 1640.4 yards) of home. These variables reflect the distances that an average adult could walk to in $5 \mathrm{~min}$ and $15 \mathrm{~min}$, respectively (i.e., $\sim 6 \mathrm{~km} / \mathrm{h}$ ).

\section{Destination mix}

Three calculated cumulative opportunity measures represented destination mix (Guy, 1983; Handy and Clifton, 2001a; Handy and Neimeier, 1997). The first measure included a total count of different types of destinations within 400 and $1500 \mathrm{~m}$ of the respondent's home. The second and third measures included the total count of different types of recreational destinations (i.e., park, beach, river) and utilitarian destinations (i.e., shop, post box, convenience store, newsagent, school, bus stop, transit station), respectively, located within 400 and $1500 \mathrm{~m}$ of the respondent's home.

\section{Covariates}

Covariates included sex, age (i.e., 18-29, 30-39, 40-49, or 50-59 years), education (i.e., $<$ high school, completed high school or trade certificate, other certificate or diploma, or tertiary), number of dependent children $<18$ years (i.e., none, one, or two or more), stratification by area level social disadvantage (i.e., highest and lowest disadvantage), and body mass index (i.e., underweight $<18.5 \mathrm{~kg} / \mathrm{m}^{2}$, acceptable weight $=18.5-24.9 \mathrm{~kg} / \mathrm{m}^{2}$, overweight $=25.0-29.9 / \mathrm{m}^{2}$, or obese $\geq 30 \mathrm{~kg} / \mathrm{m}^{2}$ ). Area level social disadvantage is based on the SocioEconomic Index for Areas (SEIFA) calculated by the Australian Bureau of Statistics. The index reflects aggregate levels of income, education and employment at the census collectors district level (Australian Bureau of Statistics, 2003).

\section{Statistical analyses}

The study design resulted in clustering at the CD level. Hence generalised estimating equations (GEE) were used for binary physical activity outcome variables and generalised linear mixed models (GLMM) used for the continuous physical activity outcome variables (i.e., average minutes of physical activity in the past fortnight). Adjusted odds ratios (ORs) and $95 \%$ confidence intervals (CI) were calculated using GEE, to examine the influence of each destination on the binary outcome variables. Adjusted ORs were also calculated to examine the linear relationship between the three land-use mix variables and regular physical activity. The odds ratios were modeled for regular versus inactive and for irregular versus inactive respondents for all physical activity outcomes. The linear relationship between the mix of all destinations, utilitarian and recreational destinations and the average duration spent per fortnight on each physical activity was examined using GLMM.

Only respondents with complete physical activity and demographic data were included in the analysis $(n=1394)$. Consistent with other Australian surveys (Armstrong et al., 2000; Bull et al., 2000) and recommendations (Australian Institute for Health and Welfare, 2003), minutes of physical activity was truncated at $1680 \mathrm{~min}$. Using SAS (9.1), all analyses adjusted for the covariates and $p$ values $<0.05$ were considered statistically significant.

\section{Results}

\section{Demographics}

The sample consisted of more women (68.7\%) than men, and more respondents with no dependent children younger than 18 years $(51.2 \%)$ than those with younger children (see Table 1). The majority of respondents had acceptable weight (52.1\%). 
Table 1

Descriptive statistics for demographic characteristics and physical activity variables (Perth, Western Australia, 1995)

\begin{tabular}{|c|c|c|c|}
\hline & $N$ & $\%$ & Mean (SD) \\
\hline \multicolumn{4}{|l|}{ Sex } \\
\hline Men & 436 & 31.3 & \\
\hline Women & 958 & 68.7 & \\
\hline \multicolumn{4}{|l|}{ Age in years } \\
\hline $18-29$ & 355 & 25.5 & \\
\hline $30-39$ & 394 & 28.3 & \\
\hline $40-49$ & 386 & 27.7 & \\
\hline $50-59$ & 259 & 18.6 & \\
\hline \multicolumn{4}{|l|}{ Education } \\
\hline Less than high school & 293 & 21.0 & \\
\hline High school or trade certificate & 406 & 29.1 & \\
\hline Other certificate or diploma & 307 & 22.0 & \\
\hline Tertiary qualification & 388 & 27.8 & \\
\hline \multicolumn{4}{|l|}{ Body mass index ${ }^{1}$} \\
\hline Underweight & 182 & 13.1 & \\
\hline Acceptable & 726 & 52.1 & \\
\hline Overweight & 341 & 24.5 & \\
\hline Obese & 129 & 9.2 & \\
\hline \multicolumn{4}{|l|}{ Children $<18$ years } \\
\hline None & 714 & 51.2 & \\
\hline One & 233 & 16.7 & \\
\hline Two or more & 447 & 32.1 & \\
\hline \multicolumn{4}{|l|}{ Socioeconomic status } \\
\hline Advantaged & 719 & 51.6 & \\
\hline Disadvantaged & 675 & 48.4 & \\
\hline \multicolumn{4}{|l|}{ Walked for transport } \\
\hline Regular & 558 & 40.0 & $190.68(196.29)^{2,3}$ \\
\hline Irregular & 394 & 28.3 & $68.35(111.25)^{2,3}$ \\
\hline None & 442 & 31.7 & \\
\hline \multicolumn{4}{|l|}{ Walked for recreation } \\
\hline Regular & 607 & 43.5 & $297.40(240.04)^{2,3}$ \\
\hline Irregular & 386 & 27.7 & $94.30(135.61)^{2,3}$ \\
\hline None & 401 & 28.8 & \\
\hline \multicolumn{4}{|l|}{ Vigorous activity } \\
\hline Regular & 492 & 35.3 & $407.45(317.10)^{2,3}$ \\
\hline Irregular & 311 & 22.3 & $122.69(121.64)^{2,3}$ \\
\hline None & 591 & 42.4 & \\
\hline
\end{tabular}

None $=$ no physical activity reported at either survey; Irregular $=$ physical activity reported at either survey but not both; Regular=physical activity reported at both surveys; ${ }^{1}$ Respondents $(n=16)$ with missing BMI data not shown but included in analysis; ${ }^{2}$ minutes/fortnight; ${ }^{3}$ regular with significantly more minutes than irregular participants $(p<0.01)$.

Over one-half of respondents walked for transport (regular= $40.0 \%$ and irregular $=28.3 \%$ ), walked for recreation (regular $=$ $43.5 \%$ and irregular $=27.7 \%$ ), or participated in vigorous physical activity (regular $=35.3 \%$ and irregular $=22.3 \%$ ). Based on responses to both surveys, the mean fortnightly duration walking for transport was $140.05( \pm 176.96)$ min, walking for recreation was $207.44( \pm 224.69) \mathrm{min}$ and vigorous physical activity was $297.17( \pm 294.19) \mathrm{min}$. Minutes of walking for recreation, walking for transport and vigorous physical activity were significantly higher among regular compared with irregular participators $(p<0.01)$.

\section{Distance to destinations}

A high proportion of respondents lived within $400 \mathrm{~m}$ of a bus stop (79.3\%) or post box (41.7\%) (Table 2). Few homes were within $400 \mathrm{~m}$ of a beach $(0.4 \%)$ or river $(0.6 \%)$. All or most respondents lived within $1500 \mathrm{~m}$ of a bus stop (100\%), a park $(99.1 \%)$ or a post box $(99.1 \%)$. Because too few respondents lived within $400 \mathrm{~m}$ of beaches and rivers and too many within $1500 \mathrm{~m}$ of bus stops and post boxes, odds ratios for each destination could not be calculated.

After adjustment, residing within $400 \mathrm{~m}$ of a convenience store (OR 1.63, CI 1.12, 2.37), bus stop (OR 1.66, CI 1.17, 2.37 ), post box (OR 2.26, CI 1.68, 3.05), shopping mall (OR 2.90 , CI 1.80, 4.68), newsagent (OR 3.09, CI 1.92, 4.94) or transit station (OR 5.00, CI 1.18, 21.25) was significantly associated with regular walking for transport (see Table 3). Residing within $400 \mathrm{~m}$ of a convenience store (OR 1.48, CI $1.00,2.20$ ), bus stop (OR 1.51, CI 1.06, 2.15), post box (OR $1.60, \mathrm{CI} 1.18,2.19)$ or shopping mall (OR 2.20, CI 1.15, 4.18) was also significantly associated with irregular walking for transport. In addition residing within $400 \mathrm{~m}$ of a shopping mall was associated with participation in irregular (OR 1.86, CI 1.08, 3.21 ), but not regular, walking for recreation. No destinations located within $400 \mathrm{~m}$ were found to be associated with regular vigorous physical activity.

After adjusting for the covariates, residing within $1500 \mathrm{~m}$ of destinations including schools (OR 1.75, CI 1.28, 2.39), convenience stores (OR 1.89, CI 1.26, 2.84), shopping malls (OR 2.07, CI 1.43, 3.00), newsagents (OR 2.20, CI 1.60, 3.03), and transit stations (OR 2.38, CI 1.67, 3.39) was significantly associated with regular walking for transport. Residing within $400 \mathrm{~m}$ of a transit station (OR 1.54, CI 1.11, 2.14), newsagent (OR 1.66, CI 1.26, 2.18) or convenience store (OR 1.88, CI $1.37,2.56)$ was also associated with irregular walking for transport. Furthermore, having a transit station located within $1500 \mathrm{~m}$ was also positively associated with regular walking for recreation (OR 1.50, CI 1.09, 2.05), while having a beach within $1500 \mathrm{~m}$ was positively associated with irregular walking for recreation (OR 1.97, CI 1.01, 3.83) and regular vigorous physical activity (OR 1.93, CI 1.20, 3.13).

\section{Destination mix}

Destination mix was lower for the $400 \mathrm{~m}$ buffer compared with the $1500 \mathrm{~m}$ buffer, and for recreational compared with

Table 2

Descriptive statistics for destination variables (Perth, Western Australia, 1995)

\begin{tabular}{llcc}
\hline Destination & $\begin{array}{l}\text { Respondents with } \\
\text { destination data }(n)\end{array}$ & $\begin{array}{l}\text { Respondents with } \\
\text { destination within } \\
400 \mathrm{~m} \mathrm{( \% )}\end{array}$ & $\begin{array}{l}\text { Respondents with } \\
\text { destination within } \\
1500 \mathrm{~m} \mathrm{( \% )}\end{array}$ \\
\hline Beach & 1394 & 0.4 & 7.5 \\
Park & 1394 & 22.2 & 99.1 \\
River & 1391 & 0.6 & 7.8 \\
School & 1391 & 7.4 & 61.3 \\
Post box & 1380 & 41.7 & 99.1 \\
Bus stop & 1284 & 79.3 & 100.0 \\
Transit station & 1391 & 1.5 & 30.8 \\
Convenience store & 1391 & 22.7 & 74.4 \\
Newsagent & 1391 & 13.2 & 63.8 \\
Shopping mall & 1394 & 8.6 & 82.6 \\
\hline
\end{tabular}

Note. $1 \mathrm{~m}=1.0936133$ yards. 
Table 3

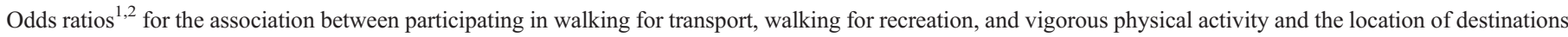
within 400 and $1500 \mathrm{~m}$ from home (Perth, Western Australia, 1995)

\begin{tabular}{|c|c|c|c|c|c|c|}
\hline & \multicolumn{2}{|l|}{ Walked for transport } & \multicolumn{2}{|l|}{ Walked for recreation } & \multicolumn{2}{|l|}{ Vigorous activity } \\
\hline & Regular vs. inactive & Irregular vs. inactive & Regular vs. inactive & Irregular vs. inactive & Regular vs. inactive & Irregular vs. inactive \\
\hline & $\mathrm{OR}(\mathrm{CI})$ & $\mathrm{OR}(\mathrm{CI})$ & $\mathrm{OR}(\mathrm{CI})$ & $\mathrm{OR}(\mathrm{CI})$ & $\mathrm{OR}(\mathrm{CI})$ & $\mathrm{OR}(\mathrm{CI})$ \\
\hline \multicolumn{7}{|l|}{ Beach } \\
\hline $\begin{array}{l}\text { Within } 400 \mathrm{~m} \\
\text { (ref.=none within } 400 \mathrm{~m} \text { ) }\end{array}$ & $\mathrm{NE}$ & $\mathrm{NE}$ & $\mathrm{NE}$ & $\mathrm{NE}$ & $\mathrm{NE}$ & $\mathrm{NE}$ \\
\hline $\begin{array}{l}\text { Within } 1500 \mathrm{~m} \\
\text { (ref.= none within } 1500 \mathrm{~m} \text { ) }\end{array}$ & $0.76(0.41,1.42)$ & $0.86(0.54,1.36)$ & $1.48(0.92,2.39)$ & $1.97(1.01,3.83)^{*}$ & $1.93(1.20,3.13)^{* *}$ & $1.20(0.71,2.03)$ \\
\hline \multicolumn{7}{|l|}{ Park } \\
\hline $\begin{array}{l}\text { Within } 400 \mathrm{~m} \\
\text { (ref.= none within } 400 \mathrm{~m} \text { ) }\end{array}$ & $0.84(0.61,1.17)$ & $1.16(0.82,1.64)$ & $1.06(0.75,1.50)$ & $1.11(0.79,1.56)$ & $1.06(0.77,1.48)$ & $1.16(0.84,1.59)$ \\
\hline $\begin{array}{l}\text { Within } 1500 \mathrm{~m} \\
\text { (ref.= none within } 1500 \mathrm{~m} \text { ) }\end{array}$ & $\mathrm{NE}$ & $\mathrm{NE}$ & NE & NE & $\mathrm{NE}$ & $\mathrm{NE}$ \\
\hline \multicolumn{7}{|l|}{ River } \\
\hline $\begin{array}{l}\text { Within } 400 \mathrm{~m} \\
\text { (ref.= none within } 400 \mathrm{~m} \text { ) }\end{array}$ & $\mathrm{NE}$ & $\mathrm{NE}$ & $\mathrm{NE}$ & $\mathrm{NE}$ & $\mathrm{NE}$ & $\mathrm{NE}$ \\
\hline $\begin{array}{l}\text { Within } 1500 \mathrm{~m} \\
\text { (ref.=none within } 1500 \mathrm{~m} \text { ) }\end{array}$ & $0.89(0.52,1.55)$ & $1.19(0.68,2.07)$ & $1.14(0.68,1.91)$ & $1.34(0.78,2.29)$ & $0.88(0.51,1.55)$ & $0.93(0.54,1.58)$ \\
\hline \multicolumn{7}{|l|}{ School } \\
\hline $\begin{array}{l}\text { Within } 400 \mathrm{~m} \\
\text { (ref.= none within } 400 \mathrm{~m} \text { ) }\end{array}$ & $0.92(0.57,1.47)$ & $0.92(0.57,1.49)$ & $0.86(0.54,1.36)$ & $0.83(0.52,1.33)$ & $0.86(0.52,1.42)$ & $0.90(0.57,1.42)$ \\
\hline $\begin{array}{l}\text { Within } 1500 \mathrm{~m} \\
\text { (ref.=none within } 1500 \mathrm{~m} \text { ) }\end{array}$ & $1.75(1.28,2.39)^{* * *}$ & $1.25(0.92,1.71)$ & $1.23(0.91,1.65)$ & $0.93(0.68,1.27)$ & $0.99(0.75,1.31)$ & $0.87(0.65,1.18)$ \\
\hline \multicolumn{7}{|l|}{ Post box } \\
\hline $\begin{array}{l}\text { Within } 400 \mathrm{~m} \\
\text { (ref.= none within } 400 \mathrm{~m} \text { ) }\end{array}$ & $2.26(1.68,3.05)^{* * *}$ & $1.60(1.18,2.19)^{* *}$ & $1.05(0.80,1.40)$ & $0.99(0.74,1.32)$ & $1.09(0.83,1.45)$ & $1.11(0.84,1.48)$ \\
\hline $\begin{array}{l}\text { Within } 1500 \mathrm{~m} \\
\text { (ref.= none within } 1500 \mathrm{~m} \text { ) }\end{array}$ & $\mathrm{NE}$ & $\mathrm{NE}$ & $\mathrm{NE}$ & $\mathrm{NE}$ & $\mathrm{NE}$ & $\mathrm{NE}$ \\
\hline \multicolumn{7}{|l|}{ Bus stop } \\
\hline $\begin{array}{l}\text { Within } 400 \mathrm{~m} \\
\text { (ref.= none within } 400 \mathrm{~m} \text { ) }\end{array}$ & $1.66(1.17,2.37)^{* *}$ & $1.51(1.06,2.15)^{*}$ & $1.18(0.83,1.67)$ & $0.94(0.67,1.31)$ & $1.24(0.90,1.71)$ & $1.33(0.92,1.92)$ \\
\hline $\begin{array}{l}\text { Within } 1500 \mathrm{~m} \\
\text { (ref.=none within } 1500 \mathrm{~m} \text { ) }\end{array}$ & $\mathrm{NE}$ & $\mathrm{NE}$ & $\mathrm{NE}$ & $\mathrm{NE}$ & $\mathrm{NE}$ & $\mathrm{NE}$ \\
\hline \multicolumn{7}{|l|}{ Transit station } \\
\hline $\begin{array}{l}\text { Within } 400 \mathrm{~m} \\
\text { (ref.= none within } 400 \mathrm{~m} \text { ) }\end{array}$ & $5.00(1.18,21.25)^{*}$ & $3.58(0.63,20.42)$ & $1.47(0.50,4.31)$ & $1.08(0.37,3.18)$ & $0.93(0.36,2.42)$ & $1.87(0.58,6.04)$ \\
\hline $\begin{array}{l}\text { Within } 1500 \mathrm{~m} \\
\text { (ref.=none within } 1500 \mathrm{~m} \text { ) }\end{array}$ & $2.38(1.67,3.39)^{* * *}$ & $1.54(1.11,2.14)^{*}$ & $1.50(1.09,2.05)^{*}$ & $1.30(0.95,1.80)$ & $0.99(0.72,1.36)$ & $1.13(0.84,1.52)$ \\
\hline \multicolumn{7}{|l|}{ Convenience store } \\
\hline $\begin{array}{l}\text { Within } 400 \mathrm{~m} \\
\text { (ref.=none within } 400 \mathrm{~m} \text { ) }\end{array}$ & $1.63(1.12,2.37)^{*}$ & $1.48(1.00,2.20)^{*}$ & $0.97(0.68,1.38)$ & $1.01(0.72,1.42)$ & $0.95(0.69,1.31)$ & $1.09(0.80,1.49)$ \\
\hline $\begin{array}{l}\text { Within } 1500 \mathrm{~m} \\
\text { (ref.=none within } 1500 \mathrm{~m} \text { ) }\end{array}$ & $1.89(1.26,2.84)^{* * *}$ & $1.88(1.37,2.56)^{* * *}$ & $1.32(0.95,1.82)$ & $1.39(0.95,2.03)$ & $0.94(0.68,1.30)$ & $1.22(0.89,1.66)$ \\
\hline \multicolumn{7}{|l|}{ Newsagent } \\
\hline $\begin{array}{l}\text { Within } 400 \mathrm{~m} \\
\text { (ref.=none within } 400 \mathrm{~m} \text { ) }\end{array}$ & $3.09(1.92,4.94)^{* * *}$ & $1.57(0.83,2.95)$ & $1.12(0.73,1.73)$ & $1.22(0.81,1.84)$ & $1.47(0.95,2.27)$ & $1.12(0.72,1.73)$ \\
\hline $\begin{array}{l}\text { Within } 1500 \mathrm{~m} \\
\text { (ref.=none within } 1500 \mathrm{~m} \text { ) }\end{array}$ & $2.20(1.60,3.03)^{* * *}$ & $1.66(1.26,2.18)^{* * *}$ & $1.31(0.97,1.76)$ & $1.14(0.83,1.57)$ & $1.10(0.83,1.46)$ & $1.00(0.75,1.33)$ \\
\hline \multicolumn{7}{|l|}{ Shopping mall } \\
\hline $\begin{array}{l}\text { Within } 400 \mathrm{~m} \\
\text { (ref.=none within } 400 \mathrm{~m} \text { ) }\end{array}$ & $2.90(1.80,4.68)^{* * *}$ & $2.20(1.15,4.18)^{*}$ & $1.58(0.94,2.66)$ & $1.86(1.08,3.21)^{*}$ & $0.96(0.61,1.53)$ & $0.71(0.44,1.18)$ \\
\hline $\begin{array}{l}\text { Within } 1500 \mathrm{~m} \\
\text { (ref.= none within } 1500 \mathrm{~m} \text { ) }\end{array}$ & $2.07(1.43,3.00)^{* * *}$ & $1.33(0.99,1.79)$ & $1.08(0.77,1.50)$ & $1.10(.076,4.58)$ & $0.92(0.66,1.29)$ & $0.96(0.70,1.33)$ \\
\hline
\end{tabular}

Note. ref. indicates the referent group. ${ }^{1}$ Derived using generalised estimating equations; ${ }^{2}$ adjusted for sex, age, area level social disadvantage, education, number of children $\leq 18$ years, and BMI; NE: not estimated odds ratios due to low proportion of destinations within buffer; $1 \mathrm{~m}=1.0936133$ yards; ${ }^{*} p<0.05, * * p<0.01$, $* * * p<0.001$.

those for utilitarian destinations (Table 4). Given the small number of respondents $(n=9)$ with no recreational destination within $1500 \mathrm{~m}$ of their home, and because only three recreational destinations were examined in this study, the variable which included the count of recreation-related only destinations within $1500 \mathrm{~m}$ was dichotomised into ' $\leq 1$ recreational destination' and ' $\geq 2$ recreational destinations'. Likewise, the majority of respondents had either 'no recreational destination 
Table 4

Odds ratios ${ }^{1,2}$ for the association between participating in walking for transport, walking for recreation, and vigorous physical activity and number of different destinations located within 400 and $1500 \mathrm{~m}$ from home (Perth, Western Australia, 1995)

\begin{tabular}{|c|c|c|c|c|c|c|}
\hline \multirow[t]{3}{*}{ Destination mix } & \multicolumn{2}{|l|}{ Walked for transport } & \multicolumn{2}{|l|}{ Walked for recreation } & \multicolumn{2}{|l|}{ Vigorous activity } \\
\hline & $\begin{array}{l}\text { Regular vs. } \\
\text { inactive }\end{array}$ & $\begin{array}{l}\text { Irregular vs. } \\
\text { inactive }\end{array}$ & $\begin{array}{l}\text { Regular vs. } \\
\text { inactive }\end{array}$ & $\begin{array}{l}\text { Irregular vs. } \\
\text { inactive }\end{array}$ & $\begin{array}{l}\text { Regular vs. } \\
\text { inactive }\end{array}$ & $\begin{array}{l}\text { Irregular vs. } \\
\text { inactive }\end{array}$ \\
\hline & OR (CI) & OR (CI) & OR (CI) & OR (CI) & $\mathrm{OR}(\mathrm{CI})$ & $\mathrm{OR}(\mathrm{CI})$ \\
\hline $\begin{array}{l}\text { Number of different types of } \\
\text { recreational and utilitarian } \\
\text { destinations within } 400 \mathrm{~m} \\
\text { (range }=0-6)\end{array}$ & $1.43(1.27,1.61)^{* * *}$ & $1.27(1.12,1.44)^{* * *}$ & $1.06(0.94,1.19)$ & $1.04(0.94,1.16)$ & $1.05(0.94,1.17)$ & $1.04(0.94,1.16)$ \\
\hline $\begin{array}{l}\text { Number of different types of } \\
\text { recreational and utilitarian } \\
\text { destinations within } 1500 \mathrm{~m} \\
\text { (range }=2-9 \text { ) }\end{array}$ & $1.41(1.26,1.58)^{* * *}$ & $1.23(1.12,1.35)^{* * *}$ & $1.16(1.06,1.27)^{* *}$ & $1.12(1.01,1.26)^{*}$ & $1.03(0.93,1.13)$ & $1.00(0.91,1.10)$ \\
\hline $\begin{array}{l}\text { Having one or more different } \\
\text { types of recreational } \\
\text { destination within } 400 \mathrm{~m}^{3} \\
\text { (ref.=having no recreational } \\
\text { destinations within } 400 \mathrm{~m} \text { ) }\end{array}$ & $0.84(0.60,1.16)$ & $1.11(0.79,1.55)$ & $1.08(0.76,1.52)$ & $1.11(0.79,1.56)$ & $1.10(0.80,1.53)$ & $1.18(0.86,1.62)$ \\
\hline $\begin{array}{l}\text { Having two or more different } \\
\text { types of recreational } \\
\text { destination within } 1500 \mathrm{~m}^{3} \\
\text { (ref.=having less than two } \\
\text { different recreational } \\
\text { destinations with } 1500 \mathrm{~m} \text { ) }\end{array}$ & $1.23(0.78,1.92)$ & $1.03(0.68,1.55)$ & $1.31(0.90,1.91)$ & $1.70(1.09,2.66)^{*}$ & $1.31(0.88,1.95)$ & $1.04(0.70,1.54)$ \\
\hline $\begin{array}{l}\text { Number of different types of } \\
\text { utilitarian destinations within } \\
400 \mathrm{~m}^{4}(\text { range }=0-6)\end{array}$ & $1.52(1.34,1.72)^{* * *}$ & $1.30(1.14,1.48)^{* * *}$ & $1.06(0.94,1.20)$ & $1.04(0.93,1.16)$ & $1.05(0.94,1.18)$ & $1.04(0.93,1.16)$ \\
\hline $\begin{array}{l}\text { Number of different types of } \\
\text { utilitarian destinations within } \\
1500 \mathrm{~m}^{4}(\text { range }=1-7)\end{array}$ & $1.49(1.32,1.67)^{* * *}$ & $1.26(1.14,1.40)^{* * *}$ & $1.17(1.05,1.29)^{* *}$ & $1.09(0.97,1.22)$ & $1.01(0.92,1.12)$ & $1.01(0.91,1.11)$ \\
\hline
\end{tabular}

Note. ref. indicates the referent group. ${ }^{1}$ Derived using generalised estimating equations; ${ }^{2}$ adjusted for sex, age, area level social disadvantage, education, number of children $\leq 18$ years, and BMI; ${ }^{3}$ includes parks, beaches, and rivers; ${ }^{4}$ includes convenience stores, newsagents, post boxes, bus stops, schools, shopping malls, and transit stations; $1 \mathrm{~m}=1.0936133$ yards; ${ }^{*} p<0.05,{ }^{* *} p<0.01,{ }^{* * *} p<0.001$.

within $400 \mathrm{~m}$ ' $(n=1077)$ or only ' 1 recreational destination within $400 \mathrm{~m}$ ' hence the variable which included the count of recreation-related destinations only within $400 \mathrm{~m}$ was dichotomised into 'no recreational destinations' and ' $\geq 1$ recreational destinations'. All other destination mix variables remained unaltered.

For each additional different type of destination (including both recreational and utilitarian destinations) within 400 and $1500 \mathrm{~m}$, the adjusted odds of regular walking for transport increased by $43 \%$ and $41 \%$ and the odds of irregular walking for transport increased by $27 \%$ and $23 \%$, respectively (Table 4 ). For each additional type of destination located within $1500 \mathrm{~m}$ the odds of regular walking for recreation increased by $16 \%$ (OR 1.16 , CI $1.06,1.27$ ), while the odds of irregular walking increased by $12 \%$ (OR 1.12, CI 1.01, 1.26). Residing with $1500 \mathrm{~m}$ of two or more different types of recreational destination was associated with irregular walking for recreation (OR 1.70, CI 1.09, 2.66). The odds increase in regular walking for transport was similar for every additional utilitarian destination located within $400 \mathrm{~m}$ (OR 1.52, CI 1.34,1.72) or within $1500 \mathrm{~m}$ (OR 1.49, CI 1.32, 1.67). Similarly, the odds increase in irregular walking for transport was similar for each additional utilitarian destination located within $400 \mathrm{~m}$ (OR 1.30, CI 1.14, 1.48) or within $1500 \mathrm{~m}$ (OR 1.26, CI 1.14, 1.40). The mix of utilitarian destinations within $1500 \mathrm{~m}$ was also positively associated with regular walking for recreation (OR 1.17, CI 1.05, 1.29).

After adjustment, for every additional recreational and utilitarian destination within 400 or $1500 \mathrm{~m}$, there was an 11.88 and $10.91 \mathrm{~min} /$ fortnight increase respectively in transport-related walking (Table 5). Furthermore, for each additional utilitarian destination within $1500 \mathrm{~m}$ walking for transport increased by $9.61 \mathrm{~min} /$ fortnight. The association between mix of utilitarian destinations within $400 \mathrm{~m}$ and minutes of walking for transport approached statistical significance $(p=0.06)$. For all behaviors, the beta coefficient increased from the 400 to $1500 \mathrm{~m}$ buffer when the mix of utilitarian and recreational destinations were examined separately, but decreased when the mix of utilitarian and recreational destinations were combined. Destination mix was not associated with time spent walking for recreation or vigorous physical activity.

\section{Discussion}

The findings of this study suggest that the proximity and mix of some types of destinations within 400 and $1500 \mathrm{~m}$ of people's homes may be more influential than others for supporting different types of physical activity (i.e., behaviorspecific). Transport-related walking appeared more influenced 
Table 5

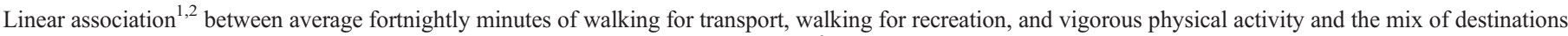
located within 400 and $1500 \mathrm{~m}$ among those reporting physical activity participation ${ }^{3}$ (Perth, Western Australia, 1995)

\begin{tabular}{|c|c|c|c|c|c|c|c|}
\hline \multirow[t]{2}{*}{ Destination } & \multirow{2}{*}{$\begin{array}{l}\text { Range of destinations } \\
\text { within buffer }\end{array}$} & \multicolumn{2}{|c|}{ Walked for transport } & \multicolumn{2}{|c|}{ Walked for recreation } & \multicolumn{2}{|c|}{ Vigorous activity } \\
\hline & & $\beta$ & Confidence interval & $\beta$ & Confidence interval & $\beta$ & Confidence interval \\
\hline $\begin{array}{l}\text { Number of different types of recreational } \\
\text { and utilitarian destinations within } 400 \mathrm{~m}\end{array}$ & $0-6$ & 11.88 & $2.90,20.86^{* *}$ & -0.75 & $-12.19,10.71$ & -9.55 & $-26.36,7.66$ \\
\hline $\begin{array}{l}\text { Number of different types of recreational } \\
\text { and utilitarian destinations within } 1500 \mathrm{~m}\end{array}$ & $2-9$ & 10.91 & $2.11,19.69^{*}$ & 3.60 & $-6.47,13.67$ & 1.16 & $-14.27,16.60$ \\
\hline $\begin{array}{l}\text { Having one or more different types of } \\
\text { recreational destinations within } 400 \mathrm{~m}^{4} \\
\text { (ref. = having no recreational destinations } \\
\text { within } 400 \mathrm{~m} \text { ) }\end{array}$ & $0-2^{6}$ & 28.58 & $-1.65,55.50$ & -2.02 & $-34.87,30.83$ & 0.24 & $-47.80,48.27$ \\
\hline $\begin{array}{l}\text { Having two or more different types of } \\
\text { recreational destination within } 1500 \mathrm{~m}^{4} \\
\text { (ref.=having less than two different } \\
\text { recreational destinations with } 1500 \mathrm{~m} \text { ) }\end{array}$ & $0-2^{6}$ & 31.96 & $-2.55,66.48$ & -7.61 & $-46.98,31.75$ & 17.06 & $-42.14,76.26$ \\
\hline $\begin{array}{l}\text { Number of different types of utilitarian } \\
\text { estinations within } 400 \mathrm{~m}^{5}\end{array}$ & $0-6$ & 9.19 & $-0.20,18.59$ & -0.83 & $-12.76,11.10$ & -10.45 & $-28.03,7.12$ \\
\hline $\begin{array}{l}\text { Number of different types of utilitarian } \\
\text { destinations within } 1500 \mathrm{~m}^{5}\end{array}$ & $1-7$ & 9.61 & $0.34,18.88^{*}$ & 4.75 & $-5.91,15.42$ & -0.09 & $-16.42,16.23$ \\
\hline
\end{tabular}

by the presence and mix of destinations compared with walking for recreation or vigorous physical activity. Positive associations between both the proximity of destinations and land-use mix and transport-related walking have been found elsewhere (Handy and Clifton, 2001b; Hoehner et al., 2005). In the current study, the presence of convenience stores, newsagencies, and shopping centres were significant correlates of transport-related walking irrespective of network buffer size examined. Furthermore, we found few correlates of vigorous physical activity, which may be due to the limited number of relevant destinations included in these analyses. Nevertheless, a preliminary conclusion drawn from our results is that built environmental policy and practice should pay additional attention to the creation of proximal transport-related destinations in order to encourage physical activity.

The dose-response relationship between the mix of destinations and walking for transport found in this study is supported elsewhere (Hoehner et al., 2005). From a public health perspective, an ideal situation might be that everyone participates in recommended levels of physical activity. However, encouraging sedentary individuals to become active is also likely to be beneficial. Our findings suggest that increasing the mix of utilitarian destinations in neighbourhoods could encourage otherwise sedentary individuals to walk for transport, while encouraging higher levels of transport-related physical activity among already active individuals. Each additional type of utilitarian destination in the neighbourhood was associated with approximately $10 \mathrm{~min}$ more transport-related walking per fortnight. Increasing the diversity of destinations should be considered highly important in the development of new neighbourhoods and for retrofitting of existing neighbourhoods. Future research should determine what mix of neighbourhood destinations is the most effective for encouraging physical activity and how the built environment might differentially influence sedentary and already active individuals.

Significant associations between the presence of destinations and walking for recreation or vigorous physical activity were limited. The presence of parks was not associated with either recreational walking or vigorous physical activity. Other studies have also reported a lack of association between parks and recreational walking and physical activity (Duncan and Mummery, 2005, Hoehner et al., 2005). It is possible that for recreational walking the mere presence of destinations is insufficient. Giles-Corti et al. (2005) found no association between a distance-only accessibility model for public open space however, higher levels of walking were evident when models included measures of attractiveness and size. Simple measures of land-use such as proximity, destination density, and cumulative opportunities ignore quality characteristics of destinations that may contribute to greater use of destinations and consequently, increased physical activity. Both proximity and the attractiveness of destinations need to be considered when designing environments supportive of physical activity.

This study highlights technical issues that may need to be considered when deciding on the size of the neighbourhood buffer to be used. In this study examining associations between the beach, river, and transit stations within $400 \mathrm{~m}$ of respondents' homes and physical activity was inappropriate because of the (1) scarcity of respondents who had access to these destinations within $400 \mathrm{~m}$ and (2) the rarity of these destinations in the areas sampled. For these less common destinations, the $1500 \mathrm{~m}$ buffer was more appropriate. Conversely, common destinations such as parks, post boxes and bus stops, the $1500 \mathrm{~m}$ buffer was inappropriate because all or most respondents had access to at least one of these 
destinations, thereby reducing variability. Transport-related destinations are generally located closer than recreational destinations (Shriver, 1997). Therefore, smaller buffers may be required for studies of transport-related physical activity and larger buffers may be required for studies of recreational or exercise-related physical activity. Decisions about buffer size need to take into consideration the abundance or paucity of destinations and the specific physical activity of interest.

\section{Limitations}

This study has several limitations. While behavior-specific destinations were examined, the physical activities studied did not necessarily occur at these destinations. Generally, mixeduse neighbourhoods have higher population densities and greater connectivity (Frank and Engelke, 2001, Frank et al., 2003, Steiner, 1994), however, no adjustment was made for these factors. Moreover, several destinations that may be important for transport-related (i.e., cafés, offices, banks) and vigorous-intensity (i.e., pools, gymnasiums, home, and the streets) physical activity were not included. The removal of respondents with missing data and the inclusion of respondents who participated in both baseline and follow-up surveys only limit the external validity of these results. Other limitations also include the cross-sectional study design, the use of self-reported data, no adjustment for multiple comparisons, and failure to control for neighbourhood self-selection.

\section{Conclusions}

Individuals generally report 'lack of time' and 'motivation' as barriers to participating in more recreational physical activity (Dishman and Sallis, 1994; Sallis and Owen, 1999). Reducing barriers to transport-related walking trips might be one strategy for ensuring that people incorporate the recommended levels of physical activity into daily activities (Frank et al., 2003). Our finding that transport-related destinations and the mix of destinations in local neighbourhoods are positively associated with transport-related walking provides some support for this strategy. More specifically, each type of destination located in a neighbourhood could increase the time spent walking for transport by approximately $5 \mathrm{~min} / \mathrm{week}$. The relationships between vigorous physical activity and walking for recreation and proximity destinations are unclear, with additional emphasis possibly required on the type and quality of destinations. The creation of supportive physical environments, including the development of destinations within walkable distances to homes may encourage more transportrelated walking and help people achieve recommended levels of physical activity.

\section{Acknowledgments}

Mr. Gavin McCormack is supported by a Western Australian Health Promotion Foundation (Healthway) Health Promotion Research Scholarship and Dr. Billie Giles-Corti by a NHMRC/ NHF Career Development Award (Grant No. 254688).

\section{References}

Armstrong, T., Bauman, A., Davies, J., 2000. Physical activity patterns of Australian adults. Results of the 1999 National Physical Activity Survey. Australian Institute of Health and Welfare, Canberra.

Aultman-Hall, L., Roorda, M., Baetz, B., 1997. Using GIS for evaluation of neighbourhoods pedestrian accessibility. J. Urban Plan. Dev. 123, 10-17.

Australian Bureau of Statistics, 2003. Information paper. Census of population and housing. socio-economic indexes for areas Australia 2001. Australian Bureau of Statistics, Canberra.

Australian Institute for Health and Welfare, 2003. The Active Australia Survey: a guide and manual for implementation, analysis and reporting. Australian Institute for Health and Welfare, Canberra.

Bauman, A., Smith, B., Stoker, L., Bellew, B., Booth, M., 1999. Geographical influences upon physical activity participation: evidence of a 'coastal effect'. Aust. N. Z. J. Public Health 23, 322-324.

Booth, M.L., Owen, N., Bauman, A.E., Gore, C.J., 1996. Retest reliability of recall measures of leisure-time physical activity in Australian adults. Int. J. Epidemiol. 25, 153-159.

Bull, F., Milligan, R., Rosenberg, M., MacGowan, H., 2000. Physical activity levels of Western Australian adults 1999. Health Department of Western Australia and Sport and Recreation Way2Go. Western Australian Government, Perth, WA.

Cervero, R., Radisch, C., 1996. Travel choices in pedestrian versus automobile oriented neighborhoods. Trans. Policy 3, 127-141.

Dishman, R.K., Sallis, J.F., 1994. Determinants and interventions for physical activity and exercise. In: Bouchard, C., Shephard, R.J., Stephens, T. (Eds.), Physical activity, fitness, and health. Human Kinetics, Champaign, IL, pp. 214-238.

Duncan, M., Mummery, K., 2005. Psychosocial and environmental factors associated with physical activity among city dwellers in regional Queensland. Prev. Med. 40, 363-372.

Frank, L.D., 2004. Economic determinants of urban form: resulting trade-offs between active and sedentary forms of travel. Am. J. Prev. Med. 27, 146-153.

Frank, L.D., Engelke, P.O., 2001. The built environment and human activity patterns: exploring the impacts of urban form on public health. J. Plan. Lit. $16,202-218$.

Frank, L.D., Engelke, P.O., Schmid, T.L., 2003. Health and community design. The impact of the built environment on physical activity. Island Press, Washington, USA.

Giles-Corti, B., Donovan, R., 2002a. The relative influence of individual, social and physical environmental determinants of physical activity. Soc. Sci. Med. 54, 1793-1812.

Giles-Corti, B., Donovan, R., 2002b. Socioeconomic status differences in recreational physical activity levels and real and perceived access to a supportive environment. Prev. Med. 35, 601-611.

Giles-Corti, B., Donovan, R., 2003. Relative influences of individual, social environmental and physical environmental correlates of walking. Am. J. Public Health 93, 1583-1589.

Giles-Corti, B., Broomhall, M.H., Knuiman, M., et al., 2005. Increasing walking: how important is distance to, attractiveness, and size of public open space? Am. J. Prev. Med. 28, 169-176.

Guy, C.M., 1983. The assessment of access to local shopping opportunities: a comparison of accessibility measures. Environ. Plan. B, Plann. Des. 10, 219-238.

Handy, S., 1996. Understanding the link between urban form and travel behaviour. J. Plann. Educ. Res. 35?, 184-198.

Handy, S., Clifton, K.J., 2001a. Evaluating neighbourhood accessibility: possibilities and practicalities. J. Transp. Stat. 4, 67-78.

Handy, S., Clifton, K.J., 2001b. Local shopping as a strategy for reducing automobile travel. Transportation 28, 317-346.

Handy, S., Neimeier, D.A., 1997. Measuring accessibility: an exploration of issues and alternatives. Environ. Plann. A 29, 1175-1194.

Hoehner, C.M., Brennan Ramirez, L.K., Elliott, M.B., Handy, S.L., Brownson, R.C., 2005. Perceived and objective environmental measures and physical activity among urban adults. Am. J. Prev. Med. 28, 105-116.

Humpel, N., Owen, N., Leslie, E., 2002. Environmental factors associated with adults' participation in physical activity: a review. Am. J. Prev. Med. 22, $188-199$. 
Humpel, N., Owen, N., Iverson, D., Leslie, E., Bauman, A., 2004. Perceived environment attributes, residential location, and walking for particular purposes. Am. J. Prev. Med. 26, 119-125.

McCormack, G., Giles-Corti, B., Lange, A., Smith, T., Martin, K., Pikora, T., 2004. An update of recent evidence of the relationship between objective and self-report measures of the physical environment and physical activity behaviours. J. Sci. Med. Sport 7, 81-92.

Owen, N., Humpel, N., Leslie, E., Bauman, A., Sallis, J.F., 2004. Understanding environmental influences on walking: review and research agenda. Am. J. Prev. Med. 27, 67-76.

Pikora, T.J., Bull, F.C., Jamrozik, K., Knuiman, M., Giles-Corti, B., Donovan, R.J., 2002. Developing a reliable audit instrument to measure the physical environment for physical activity. Am. J. Prev. Med. 23, 187-194.

Saelens, B.E., Sallis, J.F., Black, J.B., Chen, D., 2003a. Neighborhood-based differences in physical activity: an environment scale evaluation. Am. J. Public Health 93, 1552-1558.

Saelens, B.E., Sallis, J.F., Frank, L.D., 2003b. Environmental correlates of walking and cycling: findings from the transportation, urban design, and planning literatures. Ann. Behav. Med. 25, 80-91.

Sallis, J., Owen, N., 1999. Physical activity and behavioral medicine. Sage Publications Inc.

Sallis, J.F., Hovell, M.F., Hofstetter, C.R., et al., 1990. Distance between homes and exercise facilities related to frequency of exercise among San Diego residents. Public Health Rep. 105, 179-185.

Shriver, K., 1997. Influence of environmental design on pedestrian travel behavior in four Austin neighbourhoods. Transp. Res. Rec. 1578, 64-75.

Steiner, R.L., 1994. Residential density and travel patterns: review of literature. Transp. Res. Rec. 1466, 37-43.

The Government of Western Australia, 2000. Liveable neighbourhoods. A Western Australian Government Sustainable Cities Initiative. The Government of Western Australia, Perth.

Troped, P.J., Saunders, R.P., Pate, R.R., Reininger, B., Addy, C.L., 2003. Correlates of recreational and transportation physical activity among adults in a New England community. Prev. Med. 37, 304-310. 